\title{
The effect of increasing trunk flexion during normal
}

\section{walking}

\section{Abstract}

Background: The head, arms and trunk segment constitute a large proportion of the body's mass. Therefore, small alterations in trunk inclination may affect lower limb joint moments and muscle activation patterns. Although previous research has investigated the effect of changing frontal plane inclination of the trunk, it is not clear how increasing trunk flexion will impact on the activation of the lower limb muscles.

Research question: What is the effect of independently manipulating trunk flexion angle on lower limb kinematics, moments and muscle function?

Methods: Gait analysis was carried out on 20 healthy people under four trunk flexion conditions: normal walking $(\mathrm{NW}), \mathrm{NW}-5^{\circ}, \mathrm{NW}+5^{\circ}$ and $\mathrm{NW}+10^{\circ}$. For the latter three conditions, a biofeedback approach was used to tightly control trunk flexion angle. A linear mixed model was used to investigate the effect of changing trunk flexion on joint angles, moments, and knee muscle activation.

Results: There were clear increases in hip and ankle moments as trunk flexion was increased, but no change in knee moments. The results also showed a linear increase in knee flexor muscle activity and a corresponding increase in co-contraction as trunk flexion increased. Interestingly, there was a dramatic change in the profile of hamstring activity. In the medial hamstrings, this change led to a $100 \%$ increase in activation during early stance as flexion was increased by $5^{\circ}$ from NW.

Significance: This is the first study to demonstrate a strong dependence of knee flexor muscle activity on trunk flexion. This is important as people with knee osteoarthritis have been observed to 
walk with elevated muscle activation and this has been linked to increased joint loads. It is possible that these altered muscle patterns may result from increased trunk flexion during walking.

\section{Keywords:}

Trunk flexion; thorax; hamstring; co-contraction; knee osteoarthritis; EMG 


\section{Introduction}

Taken together, the head, arms and trunk segments constitute a considerable proportion of the body's mass, with cadaver studies suggesting this could be up to $65 \%$ [1]. Thus, small deviations in the position and orientation of the trunk segment have the potential to impact on the horizontal projection of the centre of mass. Understanding trunk control is therefore of critical importance from both a clinical and biomechanical standpoint as it will impact on balance [2], has been associated with functional impairment in older people [3] and may influence joint moments $[4,5]$ and muscle function $[5,6]$.

People with knee osteoarthritis are known to walk with elevated co-contraction. These altered muscle patterns are characterised by increased hamstrings $[7,8]$ quadriceps $[9,10]$ and gastrocnemius $[11,12]$ activity during the stance phase of gait. Modelling studies have demonstrated a clear peak in joint loading during early stance [13] and also shown that elevated muscle activity, during this period, will increase contact force $[13,14]$. Other related research has shown that elevated muscle activity during the early stance period will accelerate cartilage loss [15] and increase the likelihood of progression to knee arthroplasty [16]. It is therefore critical to understand the mechanisms which underlie elevated muscle activity, in people with knee osteoarthritis, during early stance.

In a recent study, we observed that people with knee osteoarthritis walk with increased trunk flexion, compared to healthy control subjects [17]. Increased trunk flexion is likely to shift the centre of mass anteriorly and therefore has the potential to alter the position and direction of the ground reaction force relative to lower limb joint centres [18]. Such biomechanical changes may alter joint moments and muscle function and may therefore underlie, to some degree, elevated muscle activity in people with knee osteoarthritis. To gain further insight into this mechanism, it is critical that we develop an in-depth understanding of the relationships between trunk flexion angle and joint moments/muscle activation. Identifying how changes in trunk flexion could impact muscle 
function may lead to new targets for interventions which could improve clinical outcomes for people with knee osteoarthritis.

Previous researchers have sought to understand the biomechanical effect of manipulating sagittal trunk angle during walking $[18,19]$. However, these studies have typically investigated large changes in trunk angle which are not representative of normal variability across human walking. For example, Saha et al. [18] instructed healthy participants to increase trunk flexion by $25^{\circ}$ and $50^{\circ}$, observing a phase lag in the position of the hip joint and a change in the vertical ground reaction force. In another study Kluger et al. [19] studied the effects of large $\left(25-50^{\circ}\right)$ changes in upper body inclination, observing a decrease in the ankle plantarflexor moment and an increased the hip extensor moment as trunk flexion was increased. Although a useful first step, these studies do not provide insight into the effects of small changes in trunk flexion, (up to $10^{\circ}$ ) which might be more typical of the variability observed during normal human walking $[4,20]$.

To date, only one study has attempted to quantify the effect of increasing trunk flexion on muscle activation [6]. However, this study was carried out with a small number of participants $(n=5)$ and investigated the effect of simultaneously increasing trunk and knee flexion during walking. Although this study showed distinct changes in muscle activation when both trunk and knee flexion were increased, it does not provide insight into the effect of independently manipulating trunk flexion angle. Given the limitations of previous research, this study sought to investigate the biomechanical effect of independently manipulating trunk flexion during normal walking. Specifically, we sought to understand the impact on lower limb kinematics, joint moments, knee muscle activation patterns and co-contraction. 


\section{Methods}

A total of 20 young healthy people (11 male) participated in this study. Subjects were excluded if they had experienced lower limb pain or back pain within the last 6 months or had been diagnosed with any neurological disease. The mean (SD) age of the participants was 26 (7) years old, mass $66.1(8.0) \mathrm{kg}$, height $1.70(0.07) \mathrm{m}$ and BMI $22.2(2.5) \mathrm{kg} / \mathrm{m}^{2}$. Ethical approval was obtained from the ethics committee at the University of Salford and all subjects provided written informed consent to participate in the study.

In order to investigate the effect of independently manipulating trunk flexion we performed a biomechanical walking assessment, for each participant, at four separate trunk flexion angles. For this assessment, kinematic and kinetic data were collected using an Oqus camera system (Qualisys, Sweden) $(100 \mathrm{~Hz})$ with two AMTI force plate $(1500 \mathrm{~Hz})$ embedded in the walkway. Reflective markers, attached the skin, were used to track motions of the pelvis and trunk, along with the thigh, shank and foot of one lower limb which was selected at random. Following the protocol suggested by Armand et al. [21] for tracking the thorax (trunk), a trunk segment was defined using markers placed on the greater trochanters and acromions. This segment was tracked using markers placed on the jugular notch and on the second and eighth thoracic vertebrae, a marker configuration which should not be influenced by arm motions during gait [21]. Preliminary testing, on five participants, showed a standard error of measurement of $0.9^{\circ}$ from test-retest data collected during two test sessions, separated by one week. Further details of the kinematic model are reported in [5] and in the supplementary materials.

Surface electromyography (EMG) data were collected from the same limb selected for the kinematic/kinetic data. These data were obtained for the following muscles using a Noraxon DTS system $(1500 \mathrm{~Hz})$ : vastus lateralis (VL), vastus medialis (VM), biceps femoris (BF), semitendinosus (ST), medial gastrocnemius (MG) and lateral gastrocnemius (LG). All electrodes were placed according to SENIAM guidelines [22] following skin preparation with an abrasive gel and alcohol 
wipe. Following electrode positioning, subjects were instructed to walk barefoot at a self-selected speed along a 6-metre walkway. A minimum of five successful walking trials were recorded for each participant, for which walking speed (measured using optical timing gates) was consistent (within a $5 \%$ tolerance) and full contact with the force platform was made.

Data from these normal walking trials were then processed to obtain a kinematic trajectory for trunk flexion angle relative to the laboratory frame. This processing involved low pass filtering of raw marker and force data at $12 \mathrm{~Hz}$ and $25 \mathrm{~Hz}$ respectively and the use of a six degree of freedom model, implemented using the Visual 3D software (C-Motion, Rockville, Maryland), to calculate the kinematic trajectory. Gait events were calculated by applying a $20 \mathrm{~N}$ threshold to the vertical ground reaction force data and used to time normalise the trunk flexion data to a full gait cycle. An ensemble average for trunk flexion was then calculated for all walking trials and the mean (across the gait cycle) used as that participant's trunk flexion angle during normal walking (NW). This was taken as the baseline condition.

In order to manipulate trunk flexion, it was necessary to instruct participants to consciously change their habitual trunk flexion angle during walking. To facilitate this, participants were guided through a two-stage process, first in standing and then in walking. During the first phase, participants developed a kinaesthetic awareness in standing of a $5^{\circ}$ and $10^{\circ}$ increase in trunk inclination (forward lean) and a $5^{\circ}$ decrease in trunk inclination (backwards lean). To facilitate this change in body alignment, participants were instructed to move their hip backwards/forwards in order to change upper body position. Pilot testing showed this instruction to be the most effective method of changing trunk flexion but minimising associated changes in knee angle or spinal alignment in standing. This phase of the training was implemented using a real-time feedback programme, deployed in MATLAB (The MathWorks), which visualised trunk inclination on a screen, indicating the target angles. 
Once participants could repeatably reproduce the target angle (trunk flexion) in standing without the need for visual feedback, walking trials at the three predetermined trunk flexion angles were carried out. These three conditions were: $\mathrm{NW}-5^{\circ}, \mathrm{NW}+5^{\circ}$ and $\mathrm{NW}+10^{\circ}$. The order of these conditions was selected at random and, for each condition, a minimum of 5 successful trials collected. A trial was considered successful if it was within $5 \%$ of the normal walking speed, appropriate contact was made with the force platform and if the mean (across the gait cycle) was within $2^{\circ}$ of the target trunk angle. After each trial, the Visual 3D software was used to calculate trunk angle and verbal feedback provided to enable participants to adjust trunk angle as appropriate.

Following the walking trials, reference data from a maximum voluntary isometric contraction (MVIC) were collected for each muscle group. These data were collected following the protocol suggested by Rutherford et al. [23] and which is described in an earlier publication [24] and in the supplementary materials. Three separate tests, of 5 seconds duration, were recorded for each of the three muscle groups with a 60 second rest between contractions. To process the MVIC data, a high pass filter $(2 \mathrm{OHz})$ was applied after which each signal was rectified and a linear envelop $(6 \mathrm{~Hz})$ created. A 0.1s moving window algorithm [9] was then applied to the linear envelope after which a maximum value was identified for each trial. The dynamic EMG was processed in a similar way, with high pass filtering $(2 \mathrm{OHz})$, followed by rectification and creation of a linear envelope $(6 \mathrm{~Hz})$. Dynamic EMG data was time normalised to stance phase and an ensemble average created for each muscle at each of the four different trunk flexion conditions. These data were then normalised by the MVIC reference value which was selected as the maximum from the three MVIC tests.

In addition to the normalised muscle activation profiles for the six individual muscles, four co-contraction activation profiles were derived. The first two profiles were obtained by summing medial/lateral hamstring and quadriceps activity and the second two profiles obtained by summing medial/lateral gastrocnemius and quadriceps activity. Previous authors have advocated using a specific co-contraction ratio [25]. However, we chose to sum medial knee flexor and knee extensor 
activity as modelling studies [26] have shown that this approach is more closely related to joint contact forces than the co-contraction ratio. Following EMG processing, kinematic trajectories for the hip, knee and ankle, along with lower limb moments were derived using the Visual 3D software using the modelling approached reported in [24] and described in the supplementary materials. All moment data were normalised by participant's body mass.

One of the primary motivators for this study was to gain insight into the potential relationship between trunk flexion and knee joint loads. Modelling studies of knee contact loads [13] have identified a point of peak load during initial stance at approximately $13 \%$ of the gait cycle, equivalent to $20 \%$ of stance phase. We therefore chose to focus on a window of $15-25 \%$ stance phase for kinematic/kinetic data. In order to define a corresponding window to characterise the EMG activity, this window was adjusted backwards by $5 \%$ of stance (approximately $30 \mathrm{~ms}$ ) to account for electromechanical delay. For the final dataset, kinematic/moment data were averaged across the window $15-25 \%$ of stance (Figure 1) and EMG/co-contraction data averaged across the window 10$20 \%$ of stance (Figures $2-4$ ). This provided a single outcome variable for each participant at each of the trunk flexion conditions for every kinematic, moment and EMG/co-contraction signal.

A linear mixed effects analysis of the relationship between the outcome variable and trunk angle was performed using R (R Core Team (2017)) and the Ime4 package [27]. Trunk angle was defined as a fixed effect, with random intercepts for each subject and by-subject random slopes for the effect of the outcome variable. No deviations from homoscedasticity or normality were seen in the residual plots, and p-values were obtained by likelihood ratio tests of two models, one with and one without the fixed effect of interest. By comparing two models, it was possible to establish whether there was a clear linear effect of trunk angle on the outcome variable of interest. 


\section{Results}

Using the two-staged biofeedback protocol, trunk angle was tightly constrained across the four different conditions (Figure 1) as demonstrated by the small standard deviations $\left(2.2-2.5^{\circ}\right)$ in trunk angle across the cohort (Table 1). Although manipulating trunk flexion angle had a clear and significant effect on hip and knee angle $(p<0.001)$, it appeared to have minimal effect on ankle angle over the period of interest (Table 1). A linear relationship between trunk angle and hip moment was observed, with an increase of $0.03 \mathrm{Nm} / \mathrm{Kg}$ for every $1^{\circ}$ increase in trunk angle across the region of interest (Table 1). This led to an increase in the hip moment of 73\% (from 23 to $49 \mathrm{Nm} / \mathrm{kg}$ ) when trunk angle was increased by $5^{\circ}$ from normal walking, an effect which appeared to be consistent across most of stance phase (Figure 1). Although trunk angle appeared to have minimal effect on the knee moment, there was a pronounced effect on ankle moment across early-mid stance (Figure 1). Again, ankle moment was observed to have a significant linear relationship $(p<0.001)$ with trunk angle across the region of interest (Table 1).

When trunk flexion angle was increased there was a dramatic rise in the activation of both semitendinosus and biceps femoris across early-mid stance (Figure 2). The activation of both hamstring muscles varied linearly with trunk angle $(p<0.001)$, with semitendinosus showing a $100 \%$ increase (from 9 to $18 \% \mathrm{MVIC}$ ) when trunk angle was increased by $5^{\circ}$ from normal walking (Table 2 ). Increases in the gastrocnemius muscles were less pronounced than the hamstring muscles but still apparent (Figure 2). Again, activity in both gastrocnemius muscles increased linearly $(p<0.001)$ over the region of interest as trunk flexion was increased (Table 2). Interestingly, there was a negligible effect of increasing trunk flexion on the two quadriceps muscles (Table 2 \& Figure 3). Increasing trunk flexion angle appeared to have a more pronounced effect on the VM-MG and VM-ST cocontraction signals than on the VL-LG and VL-BF co-contraction signals (Figure 4 \& Table 2). Nevertheless, a significant linear relationship was observed between co-contraction and trunk 
flexion $(p<0.001)$ for all signals apart from VL-LG co-contraction. For example, increasing trunk angle by $5^{\circ}$ from normal walking resulted in an increase of $23 \%$ in VM-ST co-contraction (Table 2).

\section{Discussion}

The aim of this study was to quantify the effect of independently manipulating trunk flexion on lower limb kinematics, joint moments and knee muscle activation patterns. As trunk angle was increased, the data showed an increase in the hip flexion angle as well as the hip moment and the ankle moment. Importantly, both hamstring and gastrocnemius muscles also increased with trunk flexion as did three of the four co-contraction signals. Taken together, these findings demonstrate a strong dependence of hip/ankle moments and knee flexor muscle activity on the sagittal plane inclination of the trunk.

There has been minimal research investigating the link between trunk inclination and lower limb muscle activation patterns. However, previous studies have sought to quantify the effect of trunk flexion on lower limb moments $[19,28]$. Consistent with our findings, these studies report an increase in the sagittal hip moment with a forward flexed posture. However, there were methodological differences as the investigators either did not tightly control trunk flexion angle [28] or instructed subjects to walk with an extreme forward lean of $25^{\circ}$ or $50^{\circ}$ [18]. In this current study, trunk flexion angle was tightly controlled and varied systematically by $5^{\circ}$ between each condition. The data from this study therefore provide definitive insight into changes in moments and muscle patterns which result from small changes in sagittal trunk angle in young healthy people.

In a recent study [24], we grouped healthy participants into two groups based on normal trunk flexion during walking. Comparison between the groups showed that a difference of $5^{\circ}$ in trunk flexion was associated with differences in the activation of the lateral, but not the medial, knee flexor muscles. Interestingly, the data from the current study showed a more consistent effect 
when trunk flexion was increased by $5^{\circ}$, significantly increasing both medial and lateral knee flexor muscle activity and elevating three of the four co-contraction signals. Given the use of a repeated measures design and a linear model, this current study has controlled for potential confounders. For example, precise quantification of trunk angle is difficult, as this is not a rigid segment, and this may lead to uncertainty when using a cross-sectional design to understand the association between trunk flexion and muscle patterns. However, it is possible that the contrasting findings between the two studies, reflect differences between habitual muscle patterns and muscle patterns which result from instructing participants to adopt a different trunk flexion angle over a short period of time.

Our results support the idea that, because a large proportion of the body's mass is located within the head, arms and trunk segment, small deviations in trunk flexion can have a relatively large impact on muscle patterns. This finding has important implications for musculoskeletal diseases, such as knee osteoarthritis, which are associated with elevated muscle activation, known to increase knee loads $[13,14]$. Clearly, knee osteoarthritis is a complex disease of the joint, associated with several biomechanical changes [29], such as increased knee adduction moment [30], many of which are a direct result of the disease. However, Preece et al. [17] demonstrated that people with this condition walk with a trunk flexion angle approximately $3^{\circ}$ larger than that observed in healthy people. The results of the current study demonstrate that, even this small change in postural alignment, is likely to be associated with increased knee flexor muscle activity, and therefore increased knee contact loads [13]. However, while the current study has quantified the effect of small changes in trunk flexion in healthy young people, it is not clear that the response would be the same in people with knee osteoarthritis. Nevertheless, our data motivate further research to understand the possibility of targeting postural control in order to reduce knee flexor muscle activity in people with knee osteoarthritis.

There are several strengths of the current study including the use of biofeedback to tightly control trunk flexion angle and the use of a repeated measures design. Nevertheless, limitations 
need to be highlighted. Firstly, the analysis of muscle activity was focused across a small window of the gait cycle, during early stance. This decision was made to gain insight into the effect of trunk flexion across the period corresponding to peak knee load. Although the effect of increasing trunk flexion was relatively consistent across stance phase for the hamstring muscles (Figure 1), there was more temporal variation with the gastrocnemius muscles (Figure 1). Our conclusions may therefore not extrapolate across the whole of gait cycle. A second limitation is that, although we used the same verbal instruction for all participants, it was not possible to ensure that trunk flexion was isolated at the hip joint. If this had been the case, we would have observed an LMM gradient of 1 for the hip angle (Table 1). This indicates that trunk flexion was likely achieved through flexion of the spine in addition to the hip. Nevertheless, the results still provide important insight into the effects of increasing trunk flexion during walking.

This is the first study to use a controlled experimental protocol to understand the biomechanical effect of increasing trunk flexion. The results provide important insight, showing that relatively small changes in trunk inclination can lead pronounced increases in knee flexor muscle activity and therefore co-contraction. These finding motivate future work which should seek to explore the role of trunk flexion in the altered knee muscle activation patterns which are typical of people with knee osteoarthritis.

\section{References}

[1] W.T. Dempster, Space requirements of the seated operator, geometrical, kinematic, and mechanical aspects of the body with special reference to the limbs, Michigan State Univ East Lansing, 1955.

[2] D.A. Winter, ABC (anatomy, biomechanics and control) of balance during standing and walking, Waterloo Biomechanics1995.

[3] T. Takahashi, K. Ishida, D. Hirose, Y. Nagano, K. Okumiya, M. Nishinaga, et al., Trunk deformity is associated with a reduction in outdoor activities of daily living and life satisfaction in communitydwelling older people, Osteoporosis international : a journal established as result of cooperation 
between the European Foundation for Osteoporosis and the National Osteoporosis Foundation of the USA 16(3) (2005) 273-9.

[4] S. Leteneur, C. Gillet, H. Sadeghi, P. Allard, F. Barbier, Effect of trunk inclination on lower limb joint and lumbar moments in able men during the stance phase of gait, Clinical Biomechanics 24(2) (2009) 190-195.

[5] W. Alghamdi, S.J. Preece, How does normal variability in trunk flexion affect lower limb muscle activity during walking?, Human Movement Science 72 (2020) 102630.

[6] R. Grasso, M. Zago, F. Lacquaniti, Interactions between posture and locomotion: Motor patterns in humans walking with bent posture versus erect posture, Journal of Neurophysiology 83(1) (2000) 288-300.

[7] T. Hortobagyi, L. Westerkamp, S. Beam, J. Moody, J. Garry, D. Holbert, et al., Altered hamstringquadriceps muscle balance in patients with knee osteoarthritis, Clin Biomech (Bristol, Avon) 20(1) (2005) 97-104.

[8] Rutherford, C.L. Hubley-Kozey, W.D. Stanish, Changes in knee joint muscle activation patterns during walking associated with increased structural severity in knee osteoarthritis, Journal of Electromyography and Kinesiology 23(3) (2013) 704-711.

[9] C.L. Hubley-Kozey, K.J. Deluzio, S.C. Landry, J.S. McNutt, W.D. Stanish, Neuromuscular alterations during walking in persons with moderate knee osteoarthritis, J. Electromyogr. Kinesiol. 16(4) (2006) 365-378.

[10] D.J. Rutherford, C.L. Hubley-Kozey, W.D. Stanish, M.J. Dunbar, Neuromuscular alterations exist with knee osteoarthritis presence and severity despite walking velocity similarities, Clin Biomech (Bristol, Avon) 26(4) (2011) 377-83.

[11] J.D. Childs, P.J. Sparto, G.K. Fitzgerald, M. Bizzini, J.J. Irrgang, Alterations in lower extremity movement and muscle activation patterns in individuals with knee osteoarthritis, Clinical Biomechanics 19(1) (2004) 44-49.

[12] J.L. Astephen, K.J. Deluzio, G.E. Caldwell, M.J. Dunbar, C.L. Hubley-Kozey, Gait and neuromuscular pattern changes are associated with differences in knee osteoarthritis severity levels, J Biomech 41(4) (2008) 868-76.

[13] S.C.E. Brandon, R.H. Miller, D.G. Thelen, K.J. Deluzio, Selective lateral muscle activation in moderate medial knee osteoarthritis subjects does not unload medial knee condyle, Journal of Biomechanics 47(6) (2014) 1409-1415.

[14] P. Sritharan, Y.C. Lin, S.E. Richardson, K.M. Crossley, T.B. Birmingham, M.G. Pandy, Musculoskeletal loading in the symptomatic and asymptomatic knees of middle-aged osteoarthritis patients, J Orthop Res (2016).

[15] P.W. Hodges, W. van den Hoorn, T.V. Wrigley, R.S. Hinman, K.-A. Bowles, F. Cicuttini, et al., Increased duration of co-contraction of medial knee muscles is associated with greater progression of knee osteoarthritis, Manual Therapy 21 (2016) 151-158.

[16] G.L. Hatfield, K.E. Costello, J.L. Astephen Wilson, W.D. Stanish, C.L. Hubley-Kozey, Baseline gait muscle activation patterns differ for osteoarthritis patients who undergo total knee arthroplasty 5-8 years later from those who do not, Arthritis Care Res (doi:10.1002/acr.24143) (2020).

[17] S.J. Preece, A.S. Algarni, R.K. Jones, Trunk flexion during walking in people with knee osteoarthritis, Gait \& Posture 72 (2019) 202-205.

[18] D. Saha, S. Gard, S. Fatone, The effect of trunk flexion on able-bodied gait, Gait Posture 27(4) (2008) 653-60.

[19] D. Kluger, M.J. Major, S. Fatone, S.A. Gard, The effect of trunk flexion on lower-limb kinetics of able-bodied gait, Human Movement Science 33 (2014) 395-403.

[20] K. Turcot, S. Armand, A. Lübbeke, D. Fritschy, P. Hoffmeyer, D. Suvà, Does knee alignment influence gait in patients with severe knee osteoarthritis?, Clinical biomechanics 28(1) (2013) 34-39. [21] S. Armand, M. Sangeux, R. Baker, Optimal markers' placement on the thorax for clinical gait analysis, Gait \& Posture 39(1) (2014) 147-153. 
[22] H.J. Hermens, B. Freriks, C. Disselhorst-Klug, G. Rau, Development of recommendations for SEMG sensors and sensor placement procedures, Journal of Electromyography and Kinesiology 10(5) (2000) 361-374.

[23] D.J. Rutherford, C.L. Hubley-Kozey, W.D. Stanish, Maximal voluntary isometric contraction exercises: a methodological investigation in moderate knee osteoarthritis, J Electromyogr Kinesiol 21(1) (2011) 154-60.

[24] W. Alghamdi, S. Preece, How does normal variability in trunk flexion affect lower limb muscle activity during walking?, Human Movement Science (under review) (2020).

[25] T.L. Heiden, D.G. Lloyd, T.R. Ackland, Knee joint kinematics, kinetics and muscle co-contraction in knee osteoarthritis patient gait, Clin Biomech (Bristol, Avon) 24(10) (2009) 833-41.

[26] C.R. Winby, P. Gerus, T.B. Kirk, D.G. Lloyd, Correlation between EMG-based co-activation measures and medial and lateral compartment loads of the knee during gait, Clinical Biomechanics 28(9) (2013) 1014-1019.

[27] D. Bates, M. Mächler, B. Bolker, S. Walker, Fitting Linear Mixed-Effects Models Using Ime4, Journal of Statistical Software 67 (2015) 1 - 48.

[28] C.L. Lewis, S.A. Sahrmann, Effect of posture on hip angles and moments during gait, Manual Therapy 20(1) (2015) 176-182.

[29] T.P. Andriacchi, A. Mundermann, The role of ambulatory mechanics in the initiation and progression of knee osteoarthritis, Curr Opin Rheumatol 18(5) (2006) 514-8.

[30] J.L.A. Wilson, K.J. Deluzio, M.J. Dunbar, G.E. Caldwell, C.L. Hubey-Kozey, The association between knee joint biomechanics and neuromuscular control and moderate knee osteoarthritis radiographic and pain severity, Osteoarthritis and Cartilage 19(2) (2011) 186-193. 


\section{Tables}

Table 1: Linear mixed model (LMM) analysis for lower limb moments and angles. Mean (SD) values, across the cohort, for the corresponding period of interest (Figure 1), are reported for the 4 different trunk flexion conditions ( $N W=$ normal walking). The $p$-value for the likelihood ratio test indicates whether there was a linear effect of trunk angle and if significant $(<0.001)$, LMM gradient and associated standard error (SE) are reported. The LMM gradient gives the magnitude of increase which corresponds a 10 increase in trunk angle.

\begin{tabular}{|c|c|c|c|c|c|c|}
\hline & $N W-5^{\circ}$ & $\mathrm{NW}^{\circ}$ & $N W+5^{\circ}$ & $\mathrm{NW}+10^{\circ}$ & P-value & $\begin{array}{c}\text { LMM } \\
\text { gradient } \\
\text { (SE) }\end{array}$ \\
\hline Trunk angle $\left({ }^{\circ}\right)$ & $-3.4(2.2)$ & $1.8(2.2)$ & $7.8(2.8)$ & $12.7(2.5)$ & - & - \\
\hline Hip angle $\left({ }^{\circ}\right)$ & $4.3(4.6)$ & $5.5(4.3)$ & $10.0(5.8)$ & $13.0(6.8)$ & $<0.001$ & $0.57(0.08)$ \\
\hline Knee angle $\left({ }^{\circ}\right)$ & $15.0(4.7)$ & $15.5(5.0)$ & $17.7(5.5)$ & $19.2(7.4)$ & $<0.001$ & $0.28(0.06)$ \\
\hline Ankle angle $\left({ }^{\circ}\right)$ & 7.95 (1.89) & $7.79(2.06)$ & $8.24(2.31)$ & $8.41(2.85)$ & NS & - \\
\hline $\begin{array}{c}\text { Hip moment } \\
(\mathrm{Nm} / \mathrm{kg})\end{array}$ & $0.01(0.06)$ & $0.15(0.06)$ & $0.33(0.06)$ & $0.48(0.06)$ & $<0.001$ & $0.03(0.003)$ \\
\hline $\begin{array}{c}\text { Knee moment } \\
(\mathrm{Nm} / \mathrm{kg})\end{array}$ & $0.70(0.24)$ & $0.67(0.26)$ & $0.60(0.24)$ & $0.60(0.29)$ & NS & - \\
\hline $\begin{array}{c}\text { Ankle moment } \\
(\mathrm{Nm} / \mathrm{kg})\end{array}$ & $-0.02(0.08)$ & $0.04(0.07)$ & $0.10(0.11)$ & $0.12(0.10)$ & $<0.001$ & $0.01(0.001)$ \\
\hline
\end{tabular}


Table 2: Linear mixed model (LMM) analysis for the muscle activation and co-contraction outcomes. Mean (SD) values (expressed as \%MVIC), across the cohort, for the corresponding period of interest (Figures 2-4), are reported for the 4 different trunk flexion conditions (NW=normal walking). The $p$ value for the likelihood ratio test indicates whether there was a linear effect of trunk angle and if significant $(<0.001)$, LMM gradient and associated standard error (SE) are reported. The LMM gradient gives the magnitude of increase which corresponds a 1 을 increase in trunk angle.

\begin{tabular}{|c|c|c|c|c|c|c|}
\hline & NW-5 & NW & NW+5 & NW+10 & P-value & gradient \\
& & & & & \\
(SE)
\end{tabular}




\begin{tabular}{|c|c|c|c|c|c|c|}
\hline VL-BF co- & $37(17)$ & $37(18)$ & $41(16)$ & $47(16)$ & $<0.001$ & $0.7(0.2)$ \\
contraction & & & & & \\
\hline
\end{tabular}


Figures:

Figure 1: Ensemble average (across all participants) data for the four different conditions for (a) trunk flexion, (b) hip moment, (c) knee moment and (d) ankle moment. NW denotes normal walking. The shaded regions indicate the sections of the waveforms which were averaged for the statistical analysis. Note that trunk flexion angle was averaged across the whole gait cycle. 
(a) Trunk flexion
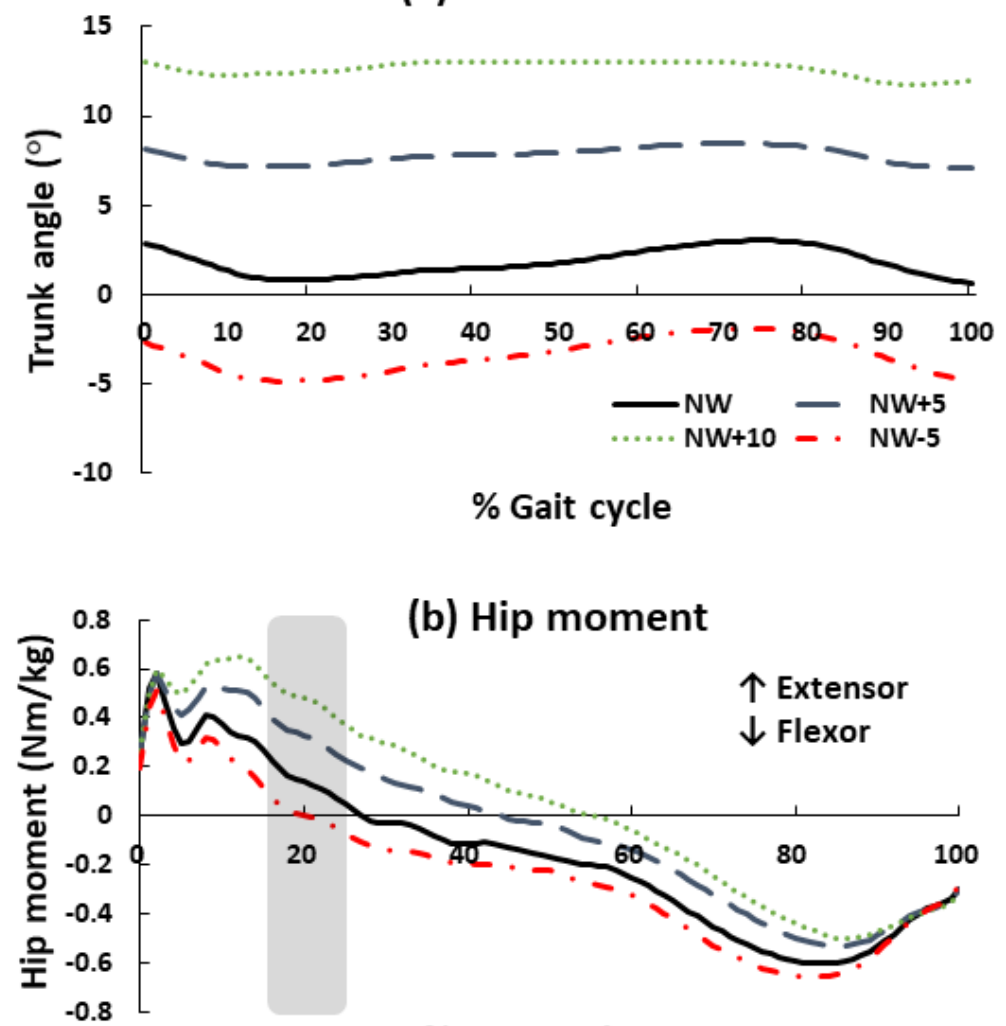

\% Stance phase

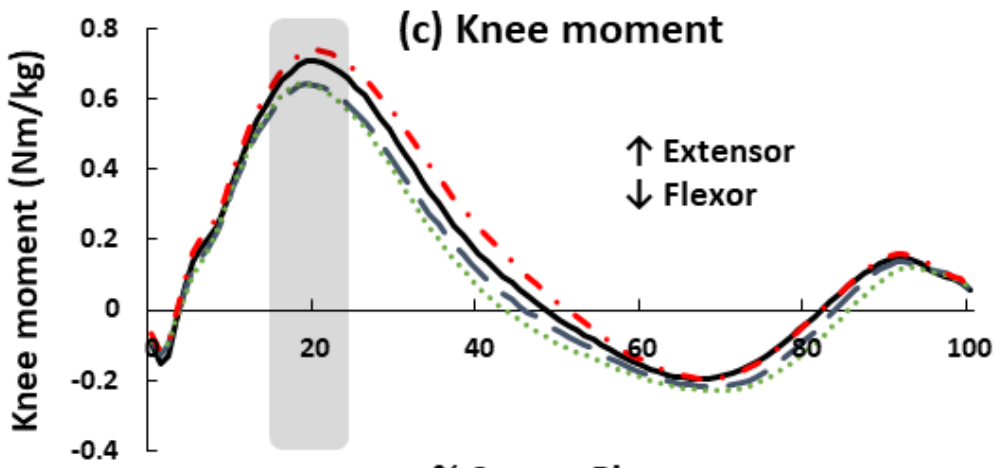

\% Stance Phase

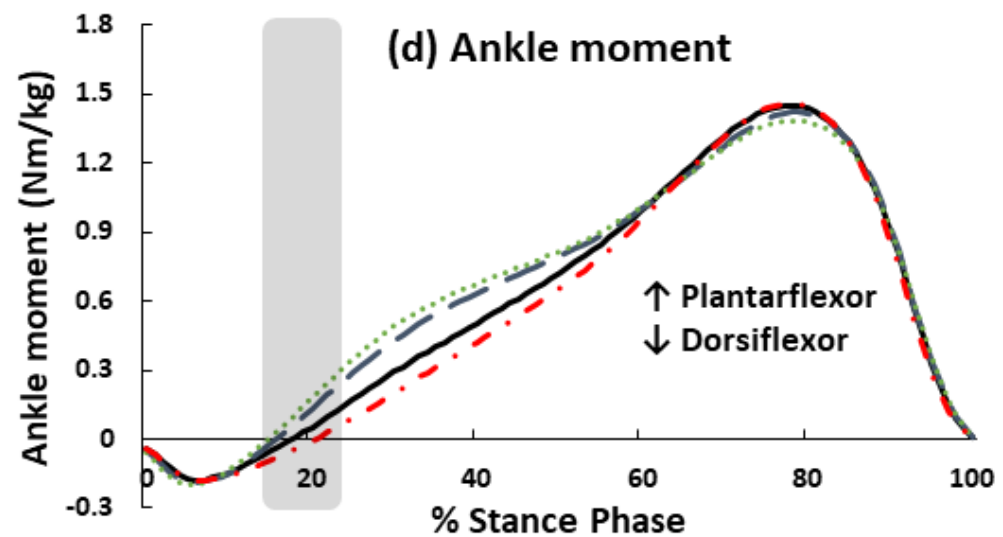


Figure 2: Ensemble average (across all participants) normalised EMG data for the four different trunk flexion conditions for (a) semitendinosus, (b) biceps femoris, (c) medial gastrocnemius and (d) lateral gastrocnemius. NW denotes normal walking. The shaded regions indicate the sections of the waveforms which were averaged for the statistical analysis. 


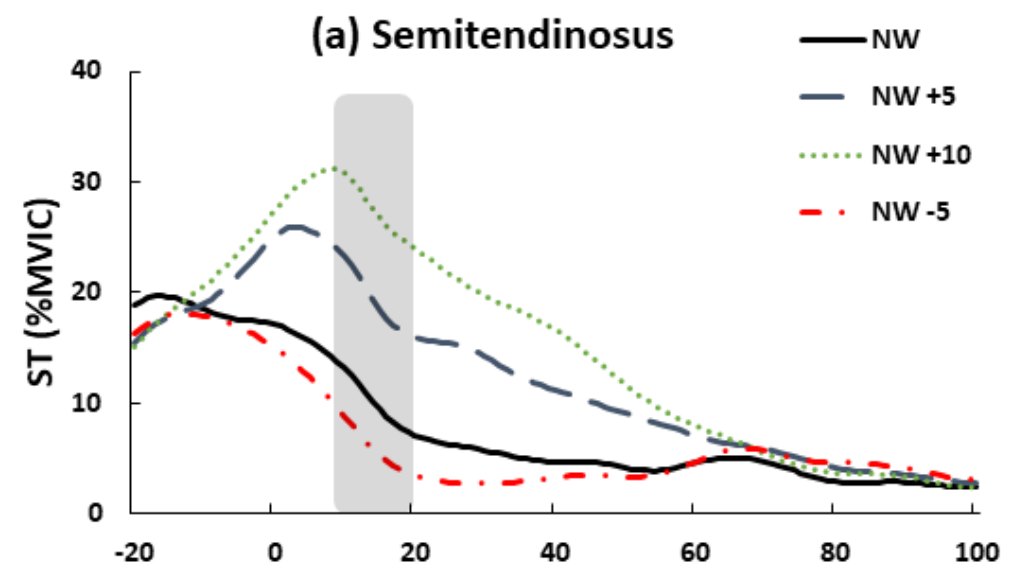

(b) Biceps femoris

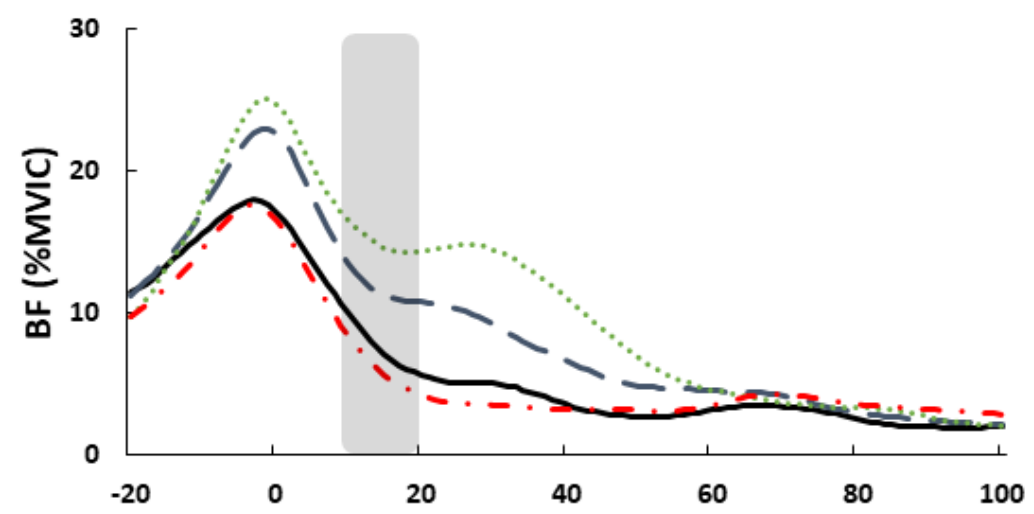

(c) Medial gastrocnemius

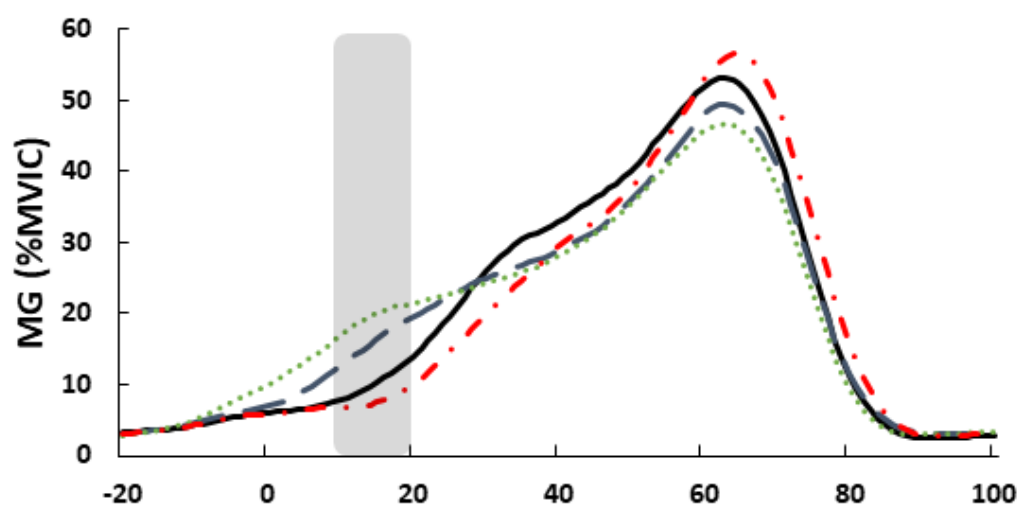

(d) Lateral gastrocnemius

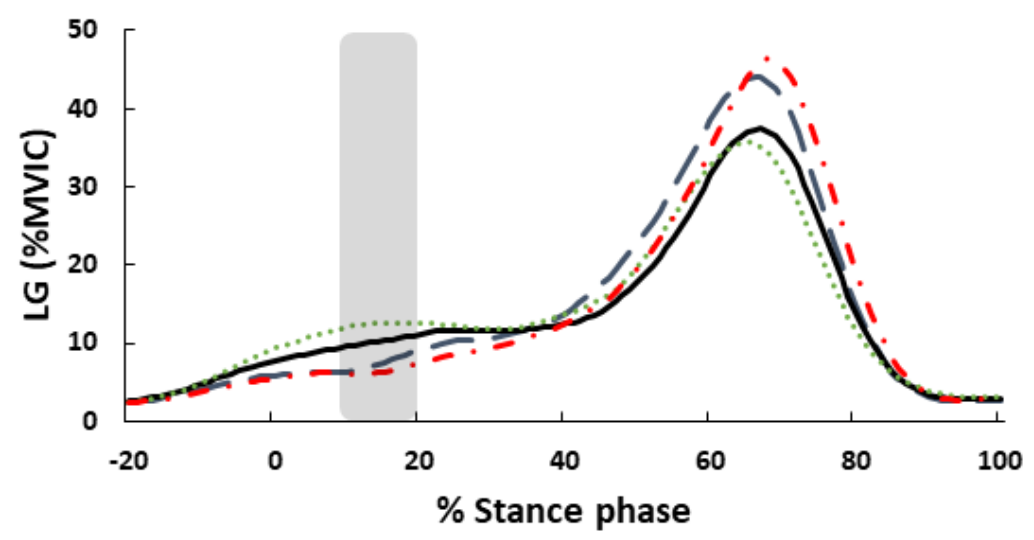


Figure 3: Ensemble average (across all participants) normalised EMG data for the two quadriceps muscle corresponding to the four different trunk flexion conditions. NW denotes normal walking. The shaded regions indicate the sections of the waveforms which were averaged for the statistical analysis. 


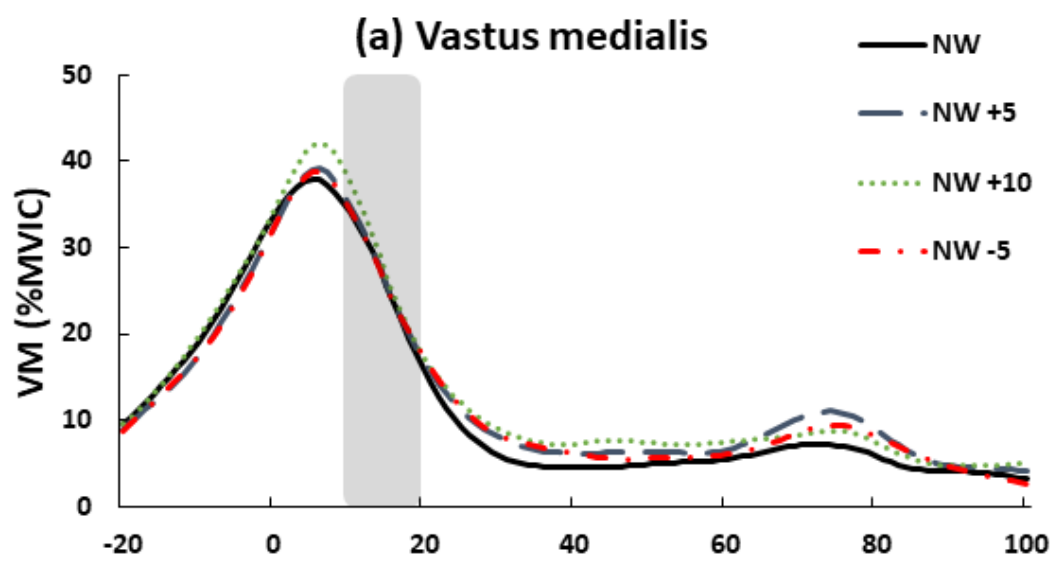

(b) Vastus lateralis

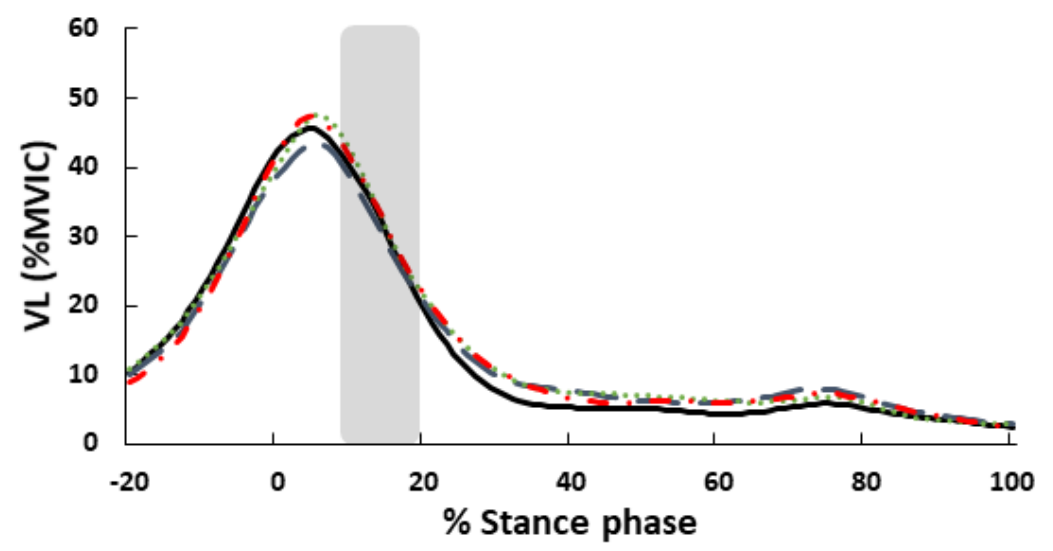


Figure 4: Ensemble average (across all participants) co-contraction signals for the four muscle pairs across different trunk flexion conditions. VM - vastus medialis, MG - medial gastrocnemius, VL vastus medialis, LG - lateral gastrocnemius, ST - semitendinosus, BF - biceps femoris. The shaded regions indicate the sections of the waveforms which were averaged for the statistical analysis. 


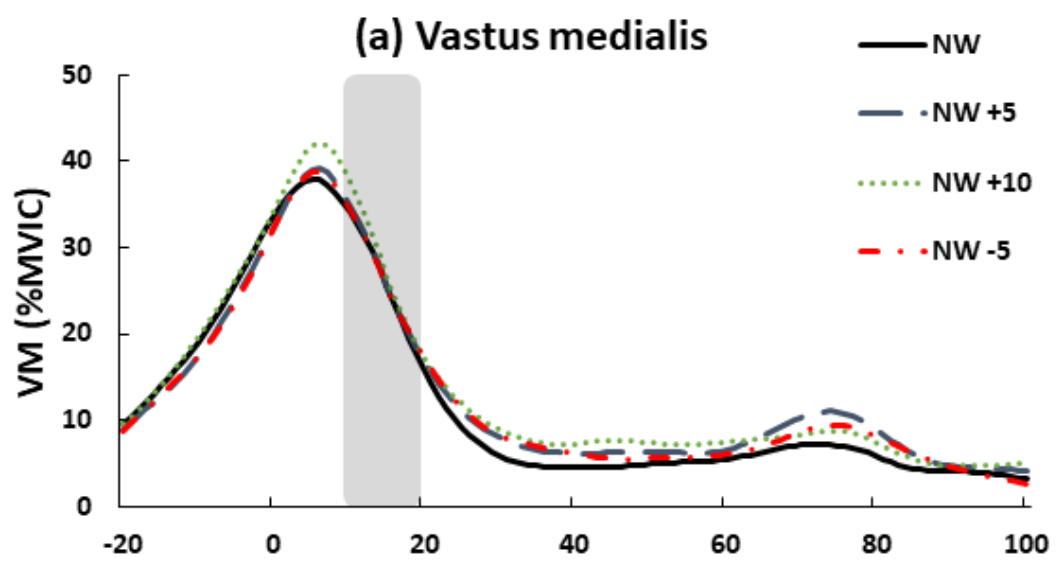

(b) Vastus lateralis

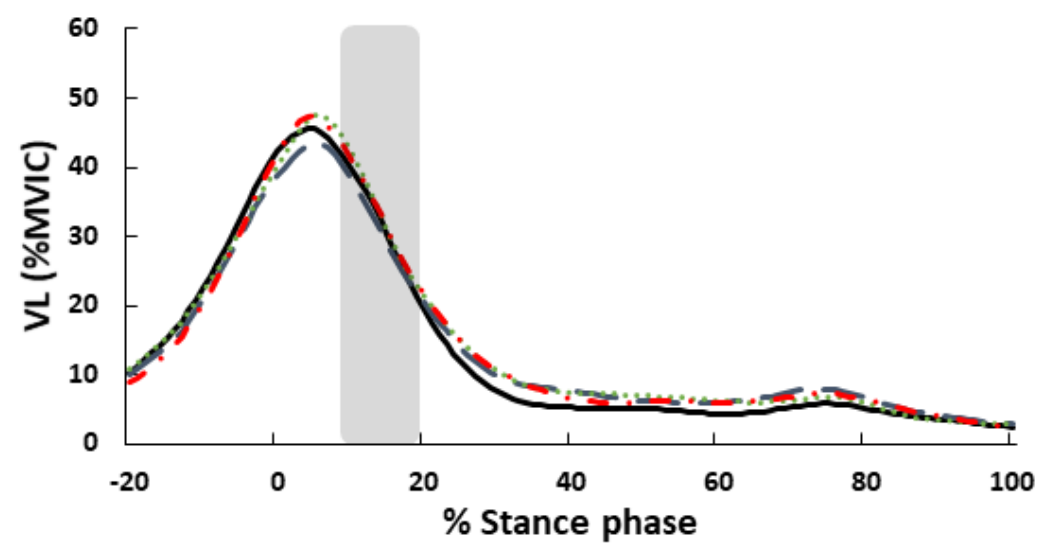

